\title{
Unusual behaviour of a retroperitoneal sarcoma: A case report and review of the literature
}

\author{
Ahmed Azzam, Abd Elrafea Elkak* \\ Department of Surgery, The Royal Hospital, Muscat, Oman
}

Received: November 9, 2016

Accepted: December 26, $2016 \quad$ Online Published: January 18, 2017

DOI: $10.5430 /$ jst.v7n1p57

URL: http://dx.doi.org/10.5430/jst.v7n1p57

\begin{abstract}
Retroperitoneal sarcomas are aggressive tumours with a poor prognosis. Staging and prognostic criteria do not always predict the behaviour of these tumours. We report a case of retroperitoneal sarcoma which did not quite follow the known prognostic criteria.
\end{abstract}

Key Words: Sarcoma, Retroperitoneal

\section{INTRODUCTION}

Retroperitoneal sarcomas are rare tumours with poor prognosis. ${ }^{[1]}$ Liposarcoma is the most frequent type followed by leiomyosarcoma. ${ }^{[2,3]}$ Malignant fibrous histiocytoma is the least frequent type. ${ }^{[2]}$ Liposarcomas are malignant tumours of mesenchymal origin which do not develop from fat but rather the tumours develop into tissues that have macroscopic and microscopic appearance similar to adipose tissue ${ }^{[2]}$ and they have a better prognosis compared to other histological subtypes. ${ }^{[4,5]}$ These tumours are generally subdivided into two major types: ${ }^{[6]} 1$ ) The infiltrative type, in which there is clear, either focal or diffuse, infiltration of visceral or muscular structures, and 2) the pushing (expansive) type, in which a viscus is totally or partially embedded into the tumour with ill-defined borders but without microscopic evidence of infiltration. Dedifferentiated liposarcomas are the most common sarcoma subtype occurring in the retroperitoneum. ${ }^{[7]}$ Although well differentiated and dedifferentiated liposarcomas have a common genetic feature associated with genomic amplification in 12q13-15, resulting in MDM2 and CDK4 over expression, ${ }^{[8]}$ the specific genetic and molecular events leading to the dissimilar behaviour are still not clear. ${ }^{[7]}$
Surgery with a negative free margin is the main line of treatment. ${ }^{[2,6]}$ The large size of these tumours and their proximity to visceral organs combined with their occurrence in the confined retroperitoneal space surrounded by critical structures make the surgical procedure complex and difficult, ${ }^{[6]}$ which means that radical wide excision, as employed with soft tissue sarcomas of other sites, may not be possible in retroperitoneal sarcoma ${ }^{[7]}$ and that is one of the reasons why retroperitoneal sarcomas have a worse prognosis than limb sarcomas. ${ }^{[2]} 75 \%$ of patients with retroperitoneal sarcoma die from local recurrence whilst systemic metastasis is the commonest form of recurrence. ${ }^{[3,9]}$ Prognosis is traditionally determined by elements of the American Joint Committee on Cancer (AJCC) staging system for sarcoma. ${ }^{[1]}$ This staging system provides prognostic factors for both extremity and retroperitoneal sarcoma based on a combination of tumour size, grade, depth, nodal involvement and the presence of distant metastasis. Other prognostic factors are multifocality and completeness of macroscopic excision. ${ }^{[7]}$ It had been shown that there are limitations to the benefit of the AJCC staging system when used as an estimate of prognosis. ${ }^{[1]}$ Tumour grade is the main variable that remains a significant

*Correspondence: Abd Elrafea Elkak; Email: aeraelkak@yahoo.co.uk; Address: Department of Surgery, The Royal Hospital, Muscat, Oman. 
predictor of survival. ${ }^{[1]}$ Significant survival benefit for patients treated with adjuvant radiotherapy or chemotherapy compared with surgical resection alone is doubtful. ${ }^{[1,6,10]}$ Abbott et al. ${ }^{[1]}$ found no significant survival benefit for patients treated with adjuvant external beam RT compared with surgical resection alone. There is, however, a lack of high quality studies to define the role of radiation in the management of patients with retroperitoneal sarcoma although radiotherapy improves local control. ${ }^{[10]}$ Most trials addressing the role of chemotherapy in the management of sarcoma were based on extremity and trunk sarcomas and the relevance of these findings to retroperitoneal sarcoma is doubtful. Therefore, the role of chemotherapy in retroperitoneal sarcoma has not been shown to significantly affect the overall or recurrence-free survival. ${ }^{[10]}$

\section{Case presentation}

A 62 years old gentleman referred from the primary health care service with bilateral inguinal hernias. On examination, the gentleman had clearly lost a significant weight and, indeed, had bilateral large inguinal hernias but on abdominal examination, he had a mass in the left side of the abdomen. Clinically, the palpable part of the mass was nodular measuring about $4 \mathrm{~cm} \times 5 \mathrm{~cm}$, and could not be traced down to the left inguinal hernia. CT scan demonstrated a predominantly fat-containing mass of heterogenous density occupying the whole left abdomen measuring $26 \mathrm{~cm} \times 16 \mathrm{~cm} \times 8 \mathrm{~cm}$ with central enhancing areas and multiple areas of enhancing septations causing mass effect on the surrounding abdominal structures most notably the left colon. The mass was of well defined outline, extending into the left inguinal hernia. Furthermore, there was superficial enhancing soft tissue mass in the left iliac fossa, related to the main fat-containing tumour, measuring around $4 \mathrm{~cm} \times 2.8 \mathrm{~cm} \times 1.7 \mathrm{~cm}$ with possible invasion of the transversus abdominis muscle. The CT showed no focal destructive bone lesions, liver metastasis or lymphadenopathy. The radiological findings were typical of retroperitoneal sarcoma (see Figure 1).

At operation, both ureters were stented. It was possible to dissect the mass safely and excise it completely. The entailed delivering the mass from the left inguinal hernia without the need for orchidectomy or resection of adjacent viscera like the left colon as it was "pushing" rather than "infiltrating" these structures but, as the mass infiltrated the anterior abdominal wall, a part of the abdominal wall was widely excised and repaired with a mesh. Postoperative course was generally uneventful, apart from intraperitoneal serous fluid collection which was readily drained percutaneously. a
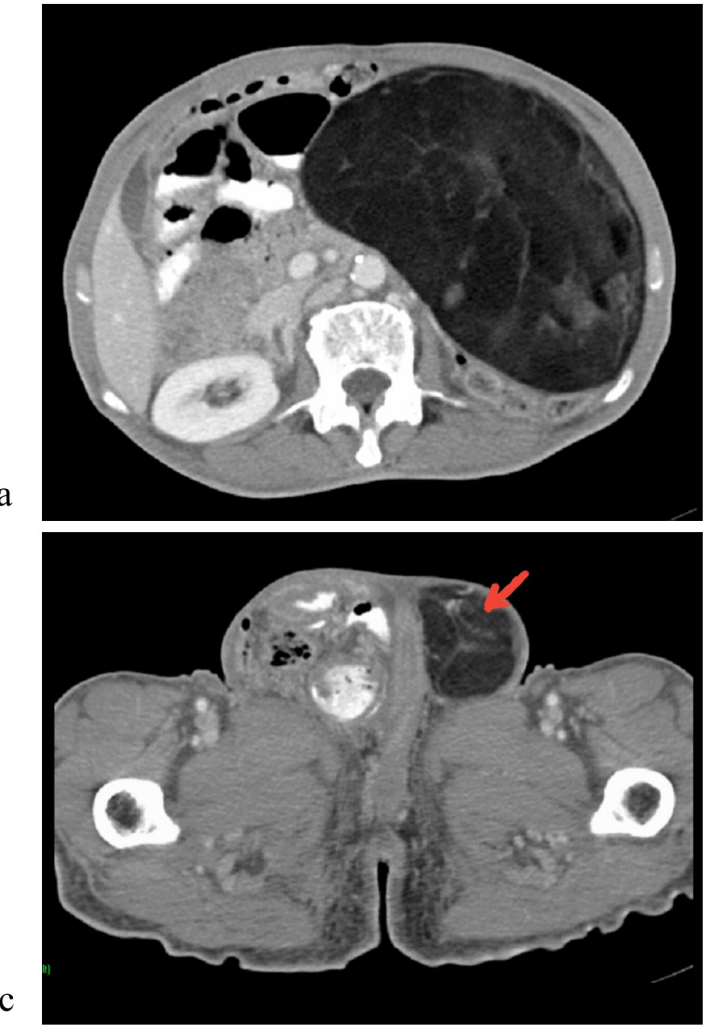

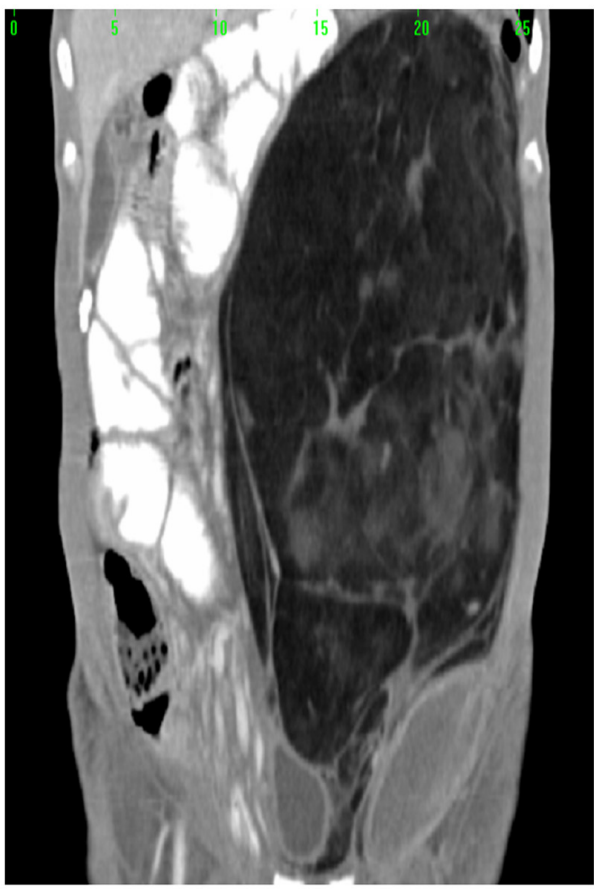

$\mathrm{b}$

Figure 1. CT of the retroperitoneal sarcoma causing mass effect on adjacent organs in (a) and (b). Bilateral inguinal hernias are seen in (c) with the tumour herniating on the left side as indicated by an arrow. 
Pathological examination demonstrated an oval shaped fibrofatty mass measuring $34 \mathrm{~cm} \times 17 \mathrm{~cm} \times 9 \mathrm{~cm}$ and weighing 2,388 grams. There was a firm area attached to the main soft tissue mass measuring $4 \mathrm{~cm} \times 3.5 \mathrm{~cm} \times 1.9 \mathrm{~cm}$. Microscopic examination showed a circumscribed and non-encapsulated tumour with features of liposarcoma. The tumour was composed of well differentiated and dedifferentiated components. The dedifferentiated component was a high grade undifferentiated pleomorphic sarcoma. This component was seen adjacent to the well differentiated tumour and was grossly identifiable (see Figure 2).

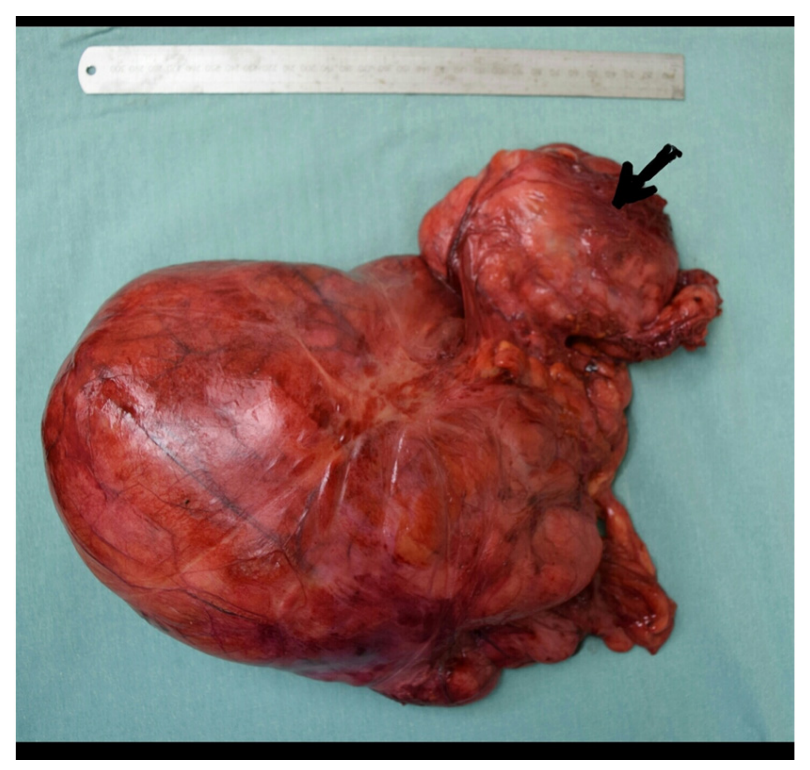

Figure 2. The liposarcoma with the clearly identifiable dedifferentiated part referred to by an arrow

Postoperative radiotherapy was considered but decided against as the patient general condition was poor and he was not expected to tolerate what would have been a large dose of radiotherapy in view of the large size of the tumour ie. the patient did not receive radiotherapy. CT performed four months after surgery showed liver and spine metastasis with possible local recurrence but there was no brain metastasis. The patient had cervical decompression and fixation of C4- D6. He continued to show generalized deterioration and passed away shortly afterwards.

\section{Discussion}

We report this case to demonstrate and discuss a few learning points. The subtle presentation with bilateral inguinal hernias is worth emphasizing. Indeed, the tumour was an incidental finding in a patient who had inguinal hernias. The extension of the mass into the left inguinal hernia raised an initial possibility that the tumour could have originated from the mesenchymal tissue of the spermatic cord but that possi-

Published by Sciedu Press bility became less likely when the tumour was dissected off the cord with no need for orchidectomy.

The tumour behaved in a mixed pushing (to the abdominal viscera) and infiltrative (to the abdominal wall). Interestingly, the dedifferentiated component was adjacent to the well differentiated tumour and grossly identifiable pathologically and that dedifferentiated component was the part that was infiltrative which is different to what had been reported by Mussi et al. ${ }^{[6]}$ It is worth noting that this dedifferentiated part was the same part palpated clinically and distinguished radiologically.

There had been a debate as to the need for multivisceral resection in retroperitoneal sarcoma. ${ }^{[6]}$ In our case here, it would have been neither required nor possible to widely resect adjacent viscera if that was contemplated not only because of the lack of capsule but also due to lack of visceral infiltration.

The early distant metastasis to the spine including the cervical spine and the liver in spite of the preoperative staging CT not demonstrating bony or liver lesions raises questions regarding how far the preoperative staging should be and whether it is justifiable to perform further imaging such as spine specific imaging or liver MRI if there is no suspicion raised on the preoperative $\mathrm{CT}$. We share the views published in a review by Tan et al. ${ }^{[10]}$ where further imaging was recommended selectively. The use of positron emission tomography (PET) scanning might have a potential in staging retroperitoneal sarcomas but that would mainly be required when there is suspicion on the initial CT. ${ }^{[2]}$ In our opinion, routine extensive imaging is not justified as the margin of benefit here, number wise, is narrow. Based on the above evidence, our patient did not have MRI, PET or bone scan as the staging CT did not show any suspicious features.

The other significance of that early recurrence and the course which was more aggressive than anticipated is how accurate the prognostic criteria are. In this case, although the tumour was a high grade as a part of it was dedifferentiated which is a poor prognostic factor, the resection, on the other hand, was complete as far as evident and possible and hence, this aggressive course was rather unexpected. Removing more "healthy" tissue would have been unnecessary and would not have improved the prognosis.

\section{Conclusion}

The behaviour of retroperitoneal sarcomas is variable and may be difficult to predict. The prognostic criteria do not apply to all cases.

\section{Conflicts of InTEREST Disclosure}

The authors declare that there is no conflict of interest statement. 


\section{REFERENCES}

[1] Abbott AM, Habermann EB, Parsons HM, et al. Prognosis for primary retroperitoneal sarcoma survivors. Cancer. 2012; 118(13): 33219. PMid:22246900. https://doi.org/10.1002/cncr. 26665

[2] Kumarasamy NA, Gayer G. Retroperitoneal sarcomas. Semin U1trasound CT MR. 2011; 32(5): 422-32. PMid:21963163. https: //doi.org/10.1053/j.sult.2011.06.002

[3] Lewis JJ, Leung D, Woodruff JM, et al. Retroperitoneal soft tissue sarcoma. Analysis of 500 patients treated and followed at a single institution. Ann Surg. 1998; 228(3): 355-65. PMid:9742918. https://doi.org/10.1097/00000658-199809000-00008

[4] Stoeckle E, Coindre JM, Bonvalot S, et al. Prognostic factors in retroperitoneal Sarcoma, A multivariate analysis of a series of 165 patients of the French Cancer Center Federation Sarcoma Group. Cancer. 2001; 92(2): 359-68. https ://doi.org/10.1002/1097 -0142(20010715) 92:2<359: : AID-CNCR1331>3.0.C0;2-Y

[5] Nathan H, Raut CP, Thornton K, et al. Predictors of survival after resection of retroperitoneal sarcoma: A population-based analysis and critical appraisal of the AJCC staging system. Ann Surg. 2009; 250(6): 970-6. PMid:19644351. https://doi.org/10.1097/SL A. 0 b013e3181b25183
[6] Mussi C, Colombo P, Bertuzzi A, et al. Retroperitoneal sarcoma: Is it time to change the surgical policy? Ann Surg Oncol. 2011; 18(8): 2136-42. PMid:21537866. https ://doi.org/10.1245/s10434 $-011-1742-z$

[7] Smith HG, Panchalingam D, Hannay JA, et al. Outcome following resection of retroperitoneal sarcoma. Br J Surg. 2015; 102(3): 1698709. PMid:26395577. https ://doi .org/10.1002/bjs. 9934

[8] Dei Tos AP, Doglioni C, Piccinin S, et al. Coordinated expression and amplification of the MDM2, CDK4, and HMGI-C genes in atypical lipomatous tumours. J Pathol. 2000; 190(5): 5316. https://doi.org/10.1002/(SICI) 1096-9896(200004) 1 $90: 5<531:$ : AID-PATH579>3.0.CO;2-W

[9] Gladdy RA, Qin LX, Moraco N, et al. Predictors of survival and recurrence in primary leiomyosarcoma. Ann Surg Oncol. 2013; 20(6): 1851-7. PMid:23354568. https : //doi.org/10.1245/s10434-0 $13-2876-y$

[10] Tan MC, Yoon SS. Surgical management of retroperitoneal and pelvic sarcomas. J Surg Oncol. 2015; 111(5): 553-61. PMid:25482329. https://doi.org/10.1002/jso. 23840 